\title{
GLIEDERUNG DES TEXTES
}

A. Allen Aussagen und Schlüssen Gemeinsames

(Kap. u. Paragr.

a) Teile des Beweises . . . . . . . . . . . . . . 1, 1-1,5

b) Die 10 Kategorien . . . . . . . . . . . . . . . 2,1

c) Teile der Aussage . . . . . . . . . . . . . . . . 2, 2-2,6

B. Zur Aussagenlogik

I. Hypothetische Aussagen
a) Allgemeines . . . . . . . . . . . . . . . .
$3,1-3,5$
b) Disjunktive Aussagen . . . . . . . . . . . . . . . . .
$4,1-4,4$
c) Konjunktive Aussagen . . . . . . . . . . . . . . . .
$4,4-4,6$
d) Implikative Aussagen . . . . . . . . . . . . . . . .
$4,6-4,7$
e) Terminologische Festsetzung zur Disjunktion . . . . . . 5, 1-5, 2

II. Hypothetische Schlüsse

a) Schlüsse aus Disjunktionen . . . . . . . . . . . . 5, 3-5,4

b) Schlüsse aus Implikationen . . . . . . . . . . . . 5, 5

III. Weitere Operationen mit Aussagen
a) Kontradiktion und Negation . . . . . . . . . . . . .
$6,1-6,2$
b) Termvertauschung und Umkehrung (unter Berücksichtigung kategorischer Aussagen). . . . . . . . . . . . . .
c) Kontraposition von Aussagen und Schlüssen . . . . . .
d) Kontraposition von Formeln, exemplifiziert an Chrysipps
Anapodeiktoi. . . . . . . . . . . . . . . . . . 6,6
$6,3-6,4$
6,5

IV. Anhang

Schlüsse aus der Quasidisjunktion . . . . . . . . . .

6,7

\section{Zur Begriffslogik}

\section{Allgemein}

a) Priorität der hypothetischen bzw. kategorischen Schlüsse

$7,1-7,3$

b) Assumption nur bei hypothetischen Schlüssen . . . . . . 7,4

c) Bildung kategorischer Schlüsse . . . . . . . . . 7, 5-7,7

d) Terminologie ................... . . 7,8-7,9 
II. Die Aristotelischen Schlüsse und ihre Ableitungen

a) der ersten Figur . . . . . . . . . . . 8, 1-4

b) der zweiten Figur . . . . . . . . . 9, 9, 9,6

c) der dritten Figur . . . . . . . . . . . . . . . 10, 1-10,8

d) Welche weiteren Schlüsse sind möglich?

1. Allgemein . . . . . . . . . . . . . 11,3

2. Nicht ausgeschöpfte Schlüsse . . . . . . . 11,4

3. Umkehrungsschlüsse (4. Figur) . . . . . . . 11, 5-11, 7

D. Beweistheorie

I. Beweise durch kategorische Schlüsse

a) Die Wichtigkeit der allgemeinen Aussage besonders für mathematische Beweise . . . . . . . . 12,1-12,5

b) Form und Terminologie für wissenschaftliche Aussagen . . 12, 5-12, 9

c) Die einzelnen Schlußfiguren und -modi als Beweise . . . 13,1-13, 6

d) Beweise in den einzelnen Kategorien . . . . . . . 13, 7-13, 12

II. Beweise durch hypothetische Schlüsse

a) Notwendig besonders in der Kategorie der Existenz . . . . 14, 1

b) Alle auf Implikationen und Disjunktionen aufgebaut . . 14, 2

c) Nicht auf Konjunktionen (gegen Chrysipp). . . . . . 14, 3-14, 6

d) Aussagenverknüpfungen und die dazugehörigen Asumptionen. ................. 14,7-14,9

e) Anzahl der hypothetischen Schlüsse. . . . . . . . 14, 10-14, 11

f) Beweise durch mehr als zweigliedrige Quasidisjunktionen . 15,1-15, 6

g) Sachverhalt und Wortlaut. Beziehungen zwischen Implikations- und Disjunktionsschlüssen mit Beispielen. . . . . . 15, 7-15, 11

E. Axiomatik

I. Der mathematischen Beweise

a) Proportionen. . . . . . . . . . . . . . 16,1-16,3

b) Summen und Differenzen ........... 16,4-16,5

c) Die Grundrechenarten. . . . . . . . . . 16,6-16,9

II. Der Beweise beliebiger Relationen

(beachte: 16,11 Umformung kategorischer in hypothetische Aussagen) . . . . . . . . . . . . 16,10-17, 3

III. Semantik und Axiomatik

a) Ist = Ist wahr . . . . . . . . . . . 17,4

b) Áquivalenz. . . . . . . . . . . . . 17,5

c) Bedeutung. ......................... 17,6-17,

d) Beziehung zwischen Bedeutung und Axiom. . . . . . 17,8-17,9

IV. Der Analogieschlu $B$

a) Allgemein ................ 18, . . . . . . . . . . . . . .

b) Mathematisch ............. 18,5-18,7

V. Klassifizierung der Beweise je nach dem Axiom . . . . 18,8

F. Utberfliissige Schluß-und Beweisformen . . . . . . . . . . 19, 1-19,6 